\title{
Las actividades de laboratorio en la formación de ingenieros: propuesta para el aprendizaje de los fenómenos de conducción eléctrica ${ }^{+*}$
}

Marta A. Pesa

Departamento de Física - Facultad de Ciencias Exactas y Tecnología Universidad Nacional de Tucumán

Facultad Regional Tucumán - Universidad Tecnológica Nacional Silvia Bravo

Departamento de Física - Facultad de Ciencias Exactas y Tecnología Universidad Nacional de Tucumán

Facultad Regional Tucumán - Universidad Tecnológica Nacional Silvia Pérez

Departamento de Física - Facultad de Ciencias Exactas y Tecnología Universidad Nacional de Tucumán

Manuel Villafuerte

Departamento de Física - Facultad de Ciencias Exactas y Tecnología Universidad Nacional de Tucumán

CONICET - Consejo Nacional de Investigaciones Científicas y Técnicas Tucumán - Argentina

\section{Resumo}

Este trabajo sintetiza los aportes de investigaciones previas realizadas en un grupo de investigación en enseñanza de las ciencias en carreras científico-tecnológicas, referidas a la resignificación de la formación experimental y el rol del laboratorio en la formación básica de los ingenieros. Tomando

Laboratory activities in engineering education: a proposal for learning electrical conduction phenomena

* Recebido: janeiro de 2014.

Aceito: maio de 2014. 
como punto de partida un modelo de aprendizaje de las ciencias sistémico donde se interrelacionan aspectos conceptuales, metodológicos, epistemológicos y actitudinales, se presenta una propuesta estructurada en base a ciclos de actividades grupales en el ámbito del laboratorio, que atienden a la progresión en la construcción del conocimiento. Se ilustra la propuesta abordando la temática de la conducción eléctrica y los modelos que describen $y$ explican los resultados experimentales. Siguiendo una metodología de trabajo integradora de aspectos experimentales y teóricos se plantean actividades a desarrollar en grupos pequeños, orientados y coordinados por el docente. Se atiende a las dificultades de los estudiantes, adoptando una dinámica recursiva que permite revisar aspectos no aprendidos o incorrectamente elaborados. Los resultados alcanzados, evaluados a través de informes grupales y evaluaciones integradoras individuales pueden considerarse satisfactorios y muestran la fertilidad del marco teórico para orientar la enseñanza hacia el desarrollo de las competencias necesarias en la formación de un estudiante de ingeniería.

Palavras-chave: Enseñanza de la física. Laboratorio. Desarrollo de competências. Conducción eléctrica.

\begin{abstract}
This paper summarizes the contributions of a previous educational research in scientific and technological careers. The research field is related to the redefinition of experimental education and the role of the laboratory in the basic Physics education of engineers. A systemic model of Sciencelearning where conceptual, methodological, epistemological and attitudinal aspects are interrelated is adopted. The proposal is structured around cycles of group activities in the laboratory context, for progressive construction of scientific knowledge. It addresses the content of electrical conduction and the models that describe and explain experimental results. The methodology integrates experimental and theoretical work. The students develop activities in small groups, guided and coordinated by the teacher. Students' difficulties are
\end{abstract}


faced by adopting a recursive teaching dynamic that allows us to review aspects unlearned or incorrectly processed. The results achieved, assessed through group reports and individual tests are satisfactory and they show the fertility of the theoretical learning framework for directing education towards conceptual and skills development, needed in the formation of an engineering student.

Keywords: Physics teaching. Laboratory. Development of skills. Electric conduction.

\section{Introducción}

Las actividades experimentales en la formación básica de científicos e ingenieros ha sido siempre una actividad reconocida en todas las universidades del mundo. Muestra de ello son las inversiones que las universidades realizan para mantener actualizado su equipamiento y procurar docentes expertos como parte de su plantel de profesores.

A pesar del esfuerzo sostenido las propuestas de enseñanza y los resultados de aprendizaje de los estudiantes no parecen satisfactorios (HODSON, 1994; TRUMPER, 2003; BAROLLI; LABURÚ; GURIDI, 2010; HOFSTEIN; LUNETTA, 2004). Los intentos de reforma han estado centrados en mejorar e incrementar las prácticas y experiencias (AGUEDA; CRUZ, 2005) más que en explicitar metas, objetivos y metodologías de trabajo. Pocas veces se cuestionan aspectos tales como: ¿qué debería aportar la actividad en el laboratorio de física a la formación de los futuros ingenieros del Siglo XXI?, ¿cuál es el objetivo del trabajo en el laboratorio? o ¿cuáles metodologías resultan más adecuadas para alcanzar esos objetivos?

Sin pretender hacer un análisis exhaustivo de los aportes del trabajo en el laboratorio a la formación de ingenieros, se pueden mencionar aquellos que señalan aspectos relevantes (CULLEN, 1996):

El laboratorio como un ámbito de desarrollo de capacidades para identificar, plantear $\mathbf{y}$ resolver problemas. Es posible plantear los trabajos prácticos de laboratorio de manera que tanto el diseño como la experimentación se articulen dentro de una investigación centrada en la resolución de problemas (GIL PÉREZ; VALDÉZ CASTRO, 1996; VALDEZ CASTRO et. al., 1999; ANDRÉ, 2004), superando la práctica habitual de prácticos tipo "receta" centrados sólo en mediciones y cálculos de algoritmos. Se trata de resignificar el laboratorio como un espacio de construcción de conocimientos y metodologías científicas donde se 
discuta la relevancia de los trabajos a desarrollar, la problemática en la que se insertan, la formulación de hipótesis y modelos, el diseño de experimentos, el análisis e interpretación de datos y resultados y la elaboración crítica de síntesis y conclusiones. Todos estos aspectos son fundamentales para formar un ingeniero capaz de generar nuevas tecnologías y/o adaptar las existentes a diferentes contextos (SALINAS, 1996).

El laboratorio pone en evidencia la influencia mutua entre ciencia y tecnología. Se pueden plantear pequeñas investigaciones a ser abordadas desde un marco teórico, proponiendo hipótesis tentativas que se prueban y contrastan. Durante ese proceso los estudiantes seleccionan y utilizan instrumentos y estrategias de diseño tecnológico (SALINAS et. al., 1998; MAIZTEGUI et. al., 2002; FERNANDEZ et. al., 2003) y a la vez, los resultados encontrados y las conclusiones de los trabajos se complementan con el análisis de las aplicaciones tecnológicas de los nuevos conocimientos. Se promueve así clarificar las relaciones mutuas entre los desarrollos científicos y tecnológicos y los procesos sociales (ACEVEDO DÍAZ, 2001; FOUREZ G., 1995).

El trabajo experimental y su estrecho vínculo con las actividades de modelado. La investigación científica y tecnológica se vale de construcciones que orientan su actividad: tanto la formulación de preguntas como las estrategias que se ponen en juego para resolverlas son guiadas por marcos teóricos de referencia que orientan su quehacer (ISLAS; PESA, 2004). El modelo representa una construcción provisoria, perfectible e idealizada de una identidad o fenómeno. La aplicabilidad del modelo a las entidades o fenómenos físicos que él representa se evalúa mediante el control experimental en un proceso dialéctico. Tal proceso comprende permanentes correcciones en el modelo (en su diseño, rango de validez, profundidad, poder explicativo). En este camino, mediante la evaluación y control de los errores experimentales, es posible salvar "la brecha" entre el modelo y la realidad.

$>$ El trabajo en el laboratorio como espacio de aprendizaje de la argumentación científica. Sin dejar de lado la importancia que revisten los contenidos conceptuales en el proceso de construcción del conocimiento científico, se está poniendo atención a los estudios de los instrumentos, criterios, modelos y reglas que los estudiantes utilizan para investigar, justificar, evaluar y valorar los conocimientos científicos. Desde esta perspectiva, adquiere especial importancia el discurso científico en el laboratorio, no sólo como enunciación de principios, leyes, teorías y conceptos, sino como discurso estructurador de las operaciones 
racionales que se utilizan para relacionar, por un lado, datos, explicaciones y conclusiones $\mathrm{y}$, por otro, los principios epistemológicos tales como la sistematicidad, la coherencia y consistencia de los razonamientos que guían las indagaciones (PESA et. al., 2013). De allí la importancia del desarrollo del lenguaje científico y de las competencias de comunicación oral y escrita (BENEITONE et. al., 2007).

El laboratorio como ámbito de trabajo en equipo. Es necesario procurar en las clases de laboratorio un rol más activo de los estudiantes. Para ello se debería brindar orientaciones mínimas para estimular en los alumnos la percepción del laboratorio como un espacio de indagación y de intercambio con sus pares, para tomar decisiones consensuadas y justificadas, en un marco de libertad intelectual. Esta concepción del trabajo de laboratorio requiere una innovación en el diseño de las actividades y también una resignificación del rol del docente.

Todos los aspectos señalados llevan a considerar la formación básica de los ingenieros como un camino para el desarrollo de competencias, entendidas como "complejas capacidades integradas para que puedan desempeñarse como sujetos responsables en diferentes situaciones y contextos, sabiendo ver, hacer, actuar y disfrutar convenientemente, evaluando alternativas, eligiendo estrategias adecuadas y haciéndose cargo de las decisiones tomadas" (BENEITONE et. al., 2007). Llevan también a preguntar ide qué manera se pueden integrar en una propuesta didáctica que resulte efectiva para lograr el desarrollo de las principales competencias?

Responder a esta pregunta implica un replanteo de la forma en que se enseña en el laboratorio y una reflexión acerca del marco de referencia desde el cual se va a proponer la metodología de trabajo, se van a diseñar las actividades para los alumnos y se van a evaluar los resultados. Desde esta perspectiva, se presenta en este trabajo el desarrollo de una propuesta específica para la enseñanza de los fenómenos de conducción eléctrica en el ámbito del laboratorio, sustentada en una visión integral y dinámica del trabajo experimental.

\section{Marco teórico de referencia}

A partir del surgimiento del constructivismo como un enfoque alternativo al aprendizaje de las ciencias se produce un cambio importante en los objetivos y propósitos del trabajo de laboratorio y las actividades se orientan así a la construcción y/o reconstrucción por parte del alumno de su propia comprensión a partir de sus conocimientos previos. Desde esta visión, se trata de actividades 
centradas en el alumno. En etapas posteriores, se reconoce la importancia de los aspectos sociales del aprendizaje y adquiere importancia tanto el papel del profesor como la interacción con los compañeros (TRUMPER, 2003).

En las últimas décadas, además, los aportes de la Psicología Cognitiva a los marcos de referencia sobre el aprendizaje de las ciencias permiten comprender mejor los procesos de construcción de conceptos, revalorizando el potencial cognitivo del trabajo colaborativo en el laboratorio y el rol del profesor para apoyar y fomentar los procesos de razonamiento en ese ámbito (ANDRÉ, 2004).

En particular, se consideran en este trabajo los aportes de la teoría de campos conceptuales de Vergnaud (2003). Dentro de este marco teórico, un concepto está formado por situaciones que le dan sentido al mismo, por el conocimiento en acción (del sujeto) que otorga significado al concepto y por representaciones simbólicas que actúan como significantes del mismo. De esta manera, un concepto no se desarrolla en una sola categoría de situaciones, sino dentro de una cierta variedad de ellas y correlativamente, una situación no se analiza con la ayuda de un solo concepto sino de varios. De acuerdo a estas premisas, una aproximación evolutiva a las competencias y las conceptualizaciones conduce inexorablemente a la consideración de una variedad de situaciones.

La idea de "campo conceptual" responde así a un planteo metodológico alrededor de las dos ideas de situación y concepto, tanto para investigar el desarrollo cognitivo en un área del conocimiento como para desarrollar propuestas didácticas tendientes al mismo (VERGNAUD, 2014).

Este marco teórico rescata e integra los aportes de Piaget y Vygotsky en cuanto considera que el aprendizaje por parte de un individuo depende necesariamente de su propia actividad, de su propio trabajo de construcción o reconstrucción de los conceptos y también de la ayuda que pueda recibir de su entorno. Justamente, una de las contribuciones más importantes de Vygotsky (1978) es el concepto de mediación como factor fundamental del desarrollo cognitivo: el aprendizaje se considera una actividad social en la cual los alumnos construyen los significados de los conceptos a través del diálogo, de discusiones y negociaciones entre compañeros y profesores.

Desde esta perspectiva, se considera que la teoría de campos conceptuales tiene potencialidad para orientar la enseñanza, en el ámbito de un laboratorio, hacia el desarrollo de las competencias necesarias en la formación de un estudiante de ingeniería.

Se presenta, entonces, una propuesta de trabajo experimental referida a conducción eléctrica en sólidos, que pretende atender a la complejidad del contenido y a la complejidad del aprendizaje a través de múltiples situaciones y 
sucesivas reutilizaciones de los contenidos conceptuales y metodológicos trabajados (Anexo I). El supuesto implícito en esta propuesta es que los estudiantes van desarrollando sus conceptualizaciones y su saber hacer a medida que van enfrentando y dominando diferentes situaciones problemáticas propuestas, en un proceso lento y complejo en el cual se producen continuamente avances y retrocesos (VERGNAUD, 2003).

Se definen de esta manera objetivos de trabajo que interrelacionan la comprensión de la naturaleza y metodología de la ciencia con la comprensión del dominio conceptual. Se intenta superar las propuestas centradas en la manipulación de instrumentos y equipamiento y el desarrollo de destrezas, hacia un laboratorio centrado en la "manipulación" y construcción colectiva de ideas científicas (HOLFSTEIN; KIND, 2012). El trabajo en el ámbito del laboratorio se integraría así como un espacio en el cual los alumnos pueden tomar decisiones y desarrollar estrategias para enfrentar las dificultades y resolver los problemas, acercándose de manera progresiva a la actividad de investigación o de desempeño profesional específico (CARRASCOSA et. al., 2006).

\section{Desarrollo de la propuesta didáctica}

\section{III.1 Estructura de las actividades}

A fin de ilustrar la metodología de trabajo propuesto se presenta en el Anexo I una guía orientadora del trabajo experimental que se propone a los estudiantes para abordar una temática fundamental en la formación básica de ingenieros: el estudio de la conducción eléctrica en sólidos. La propuesta está estructurada en base a ciclos de actividades grupales desarrolladas en grupos pequeños, que atienden a la progresión en la construcción del conocimiento y suponen una permanente interacción de los alumnos entre sí y del docente con los grupos de trabajo.

En la Fig. 1 se esquematiza la estructura de la propuesta referida al mecanismo de conducción en sólidos. El esquema ilustra la secuencia de acciones que se propone a los estudiantes. Para cada una de las situaciones problemáticas a abordar se mencionan, de arriba hacia abajo, las principales etapas involucradas en el trabajo experimental. La doble flecha entre estas etapas indica que en la investigación pueden producirse avances y retrocesos que implican revisar o repetir una etapa anterior. Se pone de manifiesto además, el carácter recursivo de estas actividades cuando al final del primer ciclo de investigación se inicia uno nuevo en torno a otro interrogante o situación problemática. A la izquierda, se 
mencionan las principales competencias que se pretende desarrollar en cada una de las etapas planteadas.

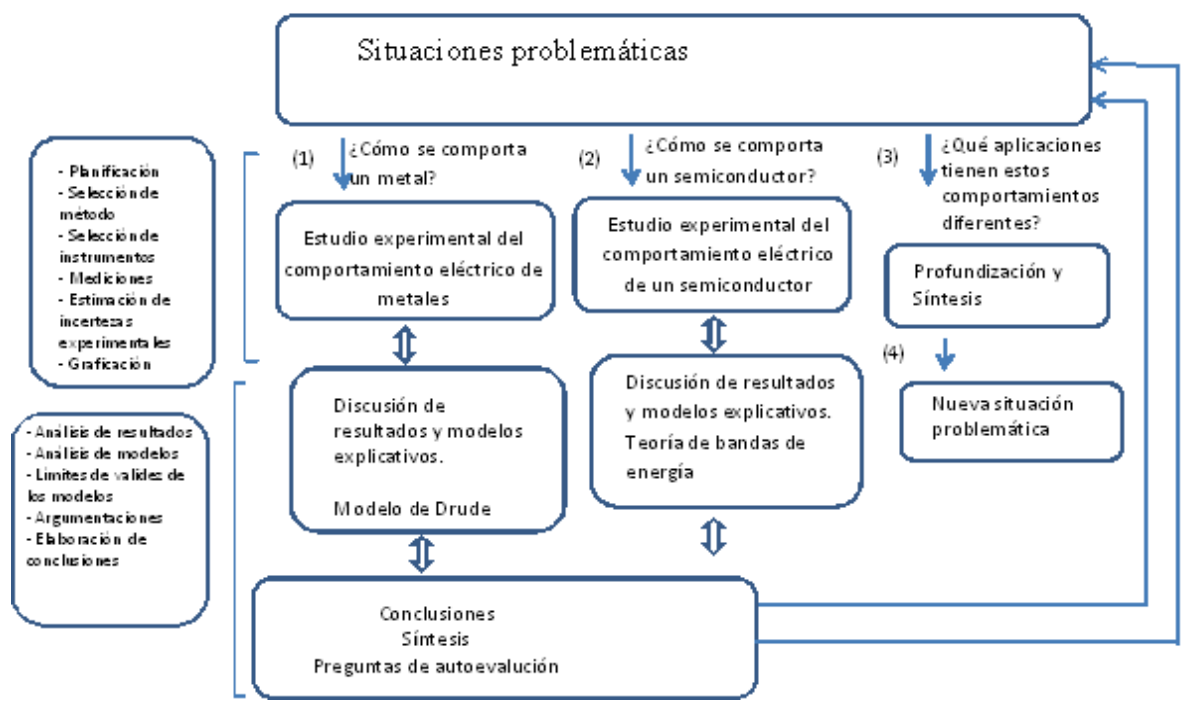

Fig. 1 - Estructura de la propuesta didáctica.

\section{III.2 Descripción de las actividades}

Al iniciar el trabajo, docente y estudiantes acuerdan los problemas a investigar y los objetivos principales del trabajo (ítems 1 y 2 del Anexo I). Este intercambio es esencial en cuanto ayuda a que el problema que inicialmente es del docente se convierta en problema de los propios estudiantes.

El primer ciclo de actividades, ¿Cómo se comporta eléctricamente un metal?, está formado por actividades introductorias, actividades de desarrollo y actividades de discusión y síntesis (ítem 3 del Anexo I). Las actividades introductorias tienen por objetivo el desarrollo de competencias relacionadas con la planificación de una experiencia de medición para estudiar la relación entre magnitudes, como ser: selección del método, selección de las características de los instrumentos, análisis de fuentes de errores sistemáticos, etc. Las actividades de desarrollo tienen por objetivo la construcción de competencias relacionadas con el aspecto operativo de una experiencia de medición: conexión de instrumentos, recolección de datos, estimación de la incerteza experimental de las mediciones, tratamiento de los datos, graficación, cálculo de parámetros, etc. Las actividades 
de discusión y síntesis tienen como principal objetivo la explicación de los comportamientos observados a través del uso de un modelo para la conducción, tal como el modelo de Drude (ASCHCROFT; MERMIN, 1976) y el análisis de los límites de validez de este modelo.

Durante el desarrollo de todas las actividades mencionadas, en especial durante las de discusión y síntesis, el profesor tiene un rol fundamental como guía y orientador a través de sugerencias y de preguntas destinadas a fomentar la discusión, la argumentación y la explicitación de las ideas de los estudiantes.

Luego de realizada la síntesis del primer ciclo de actividades el docente puede retomar los interrogantes planteados al inicio de las mismas, con ejemplos sobre algunos comportamientos de los materiales que no pueden explicarse con el modelo de Drude. Surge de esta manera la necesidad de que se hagan explícitas las limitaciones del modelo y que se identifiquen nuevas líneas de trabajo. Algunas cuestiones que se pueden abordar en esta etapa son las siguientes:

- el modelo de Drude no explica la diferencia entre aislantes y metales. Por ejemplo: el boro está arriba del aluminio en la tabla periódica, sin embargo, el boro no conduce y el aluminio es un conductor metálico.

-¿qué determina la densidad de portadores? Si todos los electrones de valencia se consideran electrones de conducción, ¿cómo se describe el comportamiento de un material aislante?

- el efecto de los iones en la dinámica del electrón y el rol de los iones como origen (fuente) de las colisiones

- el carbono en su estructura de diamante se comporta como aislante, en forma de grafito se comporta como conductor y se investiga actualmente si como grafeno puede ser superconductor.

- el modelo no explica los diferentes comportamientos con la temperatura para distintos tipos de materiales. Sin embargo, aunque no haya una dependencia explícita con la temperatura, el efecto se podría considerar en el concepto de tiempo medio de choque.

Para guiar a los alumnos en la profundización de cada uno de estos aspectos es necesario plantear una revisión crítica a la aproximación de "electrón libre" y considerar la necesidad de un modelo alternativo que pueda explicar los diferentes comportamientos.

A continuación, se retoman las situaciones problemáticas iniciales con un material de otras características (ítem 4 del Anexo) y se inicia de nuevo el ciclo de investigación acerca de un nuevo material: ¿Cómo se comporta eléctricamente un semiconductor. Las actividades de este nuevo ciclo están estructuradas de forma similar al primero, desde las actividades introductorias hasta la discusión de 
resultados y la síntesis de los mismos. En la síntesis se analizan en forma crítica los efectos de la temperatura en la determinación experimental de la relación entre tensión y corriente, abordando cuestiones como por ejemplo:

- ¿qué ocurre con la pendiente de la gráfica V-I cuando aumenta la temperatura o cuando se supera el valor de potencia máxima que puede soportar el conductor o semiconductor?

- ¿El comportamiento del semiconductor es el mismo en ambos casos? Si el comportamiento no fuera el mismo, ¿cómo se puede explicar la diferencia?

En esta etapa del desarrollo de la propuesta, el modelo de Drude resulta insuficiente para explicar estos comportamientos y debe introducirse a los alumnos hacia un análisis cualitativo del modelo de bandas (SERWAY; JEWETT, 2005). Esta apertura hacia aspectos más contemporáneos de la física en el ámbito de un laboratorio de física básica resulta muy atractiva y motivadora. Algunos cuestiones a ser discutidas en esta etapa son las siguientes:

- cómo explica el modelo de bandas los diferentes órdenes de magnitud en los valores de resistividades eléctricas de metales, semiconductores y aislantes a temperatura ambiente.

- cómo explica el modelo de bandas el comportamiento diferente de las curvas I vs $\mathrm{V}$ cuando se calienta un semiconductor o un metal

- cuáles son las limitaciones del modelo de bandas para explicar el comportamiento eléctrico de materiales con fuerte correlación electrónica (por ejemplo: superconductores).

Una vez que se han explicado los diferentes comportamientos en base a dos modelos diferentes estableciendo los límites de validez de los mismos, se puede abrir otro ciclo de profundización de los conceptos abordados, en especial el concepto de resistencia eléctrica. La discusión grupal, con la orientación del docente, sobre el uso de los distintos materiales en la tecnología, permite que los estudiantes identifiquen cuestiones abiertas para iniciar nuevas investigaciones y que elaboren el diseño de experiencias para controlar la validez de los modelos en otros aspectos, por ejemplo:

- la relación de la resistencia con la longitud y la sección de un conductor,

- la medición de la densidad electrónica utilizando el Efecto Hall clásico en el marco del modelo de Drude,

- el estudio experimental de la variación de la resistencia con la temperatura de distintos materiales y los modelos que explican los distintos comportamientos. 


\section{Resultados}

La propuesta ha sido implementada durante cinco ciclos lectivos en el ámbito de un laboratorio de física con grupos de aproximadamente 15 alumnos de la carrera licenciatura en física. Los primeros ensayos se han tomado como prueba piloto a fin de realizar los ajustes necesarios en las actividades. Se comenzó a implementar hace dos años en el ciclo básico de carreras de ingeniería con grupos de aproximadamente 60 alumnos. Cada ciclo de las actividades de la propuesta se desarrolló en dos jornadas de aproximadamente cuatro horas cada una.

Los resultados obtenidos en el último año con ambos grupos de alumnos han sido analizados cualitativamente a través de los siguientes registros: cuadernos de notas de los alumnos, informes de actividades experimentales y evaluación integradora al finalizar las actividades.

Los cuadernos de notas constituyen un espacio de registro del desarrollo del trabajo en clase de los estudiantes. Se incluyen tanto exploraciones individuales como resultados de la actividad de discusión grupal y de los consensos alcanzados. El docente supervisa durante la clase los cuadernos de notas, detectando núcleos de dificultad y/o errores conceptuales y metodológicos que deben ser revisados o corregidos. Estos registros permiten al docente evaluar en una primera etapa del trabajo la planificación de la experiencia, la selección del método, selección de instrumentos, mediciones realizadas, estimación de incertezas y registros gráficos. Y en una segunda etapa, permiten evaluar el análisis de resultados, análisis de modelos, grado de ajuste entre los resultados experimentales y el modelo teórico y análisis y control experimental de supuestos del modelo. Los registros de los cuadernos de notas constituyen para los alumnos la principal fuente de información para la elaboración del informe escrito grupal.

Los informes de las actividades experimentales son elaborados grupalmente por los estudiantes ( 2 o 3 estudiantes por grupo) y forman parte de una etapa final de síntesis y evaluación que, en general, resulta difícil y compleja a los estudiantes. Exige repensar, relacionar, reconstruir, transformar y reorganizar ideas desarrolladas en las clases, con el objetivo de elaborar un documento articulado para ser comunicado a los otros. La evaluación de los informes de los alumnos se realiza con realimentación, es decir, tienen oportunidad de corregir y completar los aspectos no satisfactorios del informe.

La evaluación integradora (Anexo II), de carácter individual, informa sobre el avance o progreso en determinado dominio o conocimiento, es decir, proporciona indicaciones de las competencias que va adquiriendo el estudiante 
(POZO; PÉREZ ECHEVERRÍA, 2009). Con este criterio, se han elaborado una serie de preguntas y problemas para evaluar algunas de esas competencias básicas al finalizar el desarrollo de la propuesta, que han sido sometidos a la revisión de pares docentes que se desempeñan en la misma asignatura. La evaluación se realiza con realimentación, es decir, está integrada al proceso de aprendizaje y su principal objetivo es formativo. Los estudiantes, tanto aprobados como desaprobados, tienen la posibilidad de rever y corregir sus errores. Los alumnos desaprobados tienen otra instancia de evaluación con una prueba similar a la del Anexo II. No solo se evalúa para promover sino para ayudar a los alumnos a alcanzar las competencias deseadas para la unidad de aprendizaje.

En este trabajo se presentan los resultados obtenidos con dos registros: los informes de la actividad y la evaluación integradora. Ambos instrumentos informan acerca de logros obtenidos al final del proceso. Los resultados del análisis de los cuadernos de notas, así como un análisis diferenciado que permita comparar logros obtenidos en la primera instancia de evaluación y de presentación de informes con los resultados finales del proceso, se reservan para un estudio posterior acerca de los procesos involucrados durante el aprendizaje y se espera que esos nuevos resultados complementen a los que se consignan en este trabajo.

\section{IV.1 Resultados del análisis de informes grupales}

La Tabla 1 muestra las competencias básicas que son evaluadas a través de los informes y las dimensiones que se han considerado en cada una de ellas. Los resultados corresponden a los logros finales de los estudiantes después de haber corregido y completado aquellos aspectos que el docente aconseja mejorar.

Para evaluar el grado de logro de cada una de las dimensiones se han clasificado las respuestas en tres categorías: Muy Bueno (MB), Bueno (B) y Regular (R), consignando los resultados obtenidos en el último ciclo lectivo con ambos grupos de alumnos. Las categorías mencionadas se pueden corresponder en algunos casos con: Siempre (S), Casi Siempre (CS) y A veces (AV). Se incluye debajo de la Tabla 1 un gráfico de barras que permite visualizar mejor los resultados y comparar los logros obtenidos en cada una de las dimensiones evaluadas (Fig. 2). 
Tabla 1 - Competencias evaluadas a través de informes grupales.

\begin{tabular}{|c|c|c|c|c|}
\hline & Dimensiones & MB & B & $\mathbf{R}$ \\
\hline \multirow{3}{*}{$\begin{array}{l}\text { Competenci } \\
\text { as para la } \\
\text { organiza- } \\
\text { ción y } \\
\text { presenta- } \\
\text { ción de las } \\
\text { ideas }\end{array}$} & $\begin{array}{l}\text { Consistencia en la estructura y } \\
\text { articulación entre las partes }\end{array}$ & $70 \%$ & $20 \%$ & $10 \%$ \\
\hline & $\begin{array}{l}\text { Explicitación clara y precisa del } \\
\text { problema a investigar, objetivos, marco } \\
\text { teórico, metodología, análisis de datos, } \\
\text { elaboración de conclusiones, aspectos } \\
\text { abiertos y referencias bibliográficas }\end{array}$ & $60 \%$ & $30 \%$ & $10 \%$ \\
\hline & Claridad y precisión en el lenguaje & $80 \%$ & $15 \%$ & $5 \%$ \\
\hline \multirow{3}{*}{$\begin{array}{l}\text { Competen- } \\
\text { cias } \\
\text { Cognitivas }\end{array}$} & $\begin{array}{l}\text { Comprensión de conceptos (tensión, } \\
\text { corriente, resistencia, resistividad) }\end{array}$ & $80 \%$ & $20 \%$ & --- \\
\hline & $\begin{array}{l}\text { Comprensión de modelos: } \\
\text { *modelo de Drude } \\
\text { *modelo simplificado y cualitativo de } \\
\text { bandas }\end{array}$ & $50 \%$ & $30 \%$ & $20 \%$ \\
\hline & $\begin{array}{l}\text { Descripción y explicación del } \\
\text { comportamiento de materiales } \\
\text { conductores }\end{array}$ & $50 \%$ & $30 \%$ & $20 \%$ \\
\hline \multirow{5}{*}{$\begin{array}{l}\text { Competen- } \\
\text { cias } \\
\text { metodológi } \\
\text { cas }\end{array}$} & $\begin{array}{l}\text { Aplicación del conocimiento teórico en } \\
\text { el diseño, realización e interpretación } \\
\text { de las experiencias }\end{array}$ & $60 \%$ & $10 \%$ & $30 \%$ \\
\hline & $\begin{array}{l}\text { Evaluación y estimación del grado de } \\
\text { ajuste entre modelos y sistemas reales }\end{array}$ & $80 \%$ & $20 \%$ & -- \\
\hline & $\begin{array}{l}\text { Argumentaciones válidas identificando } \\
\text { hipótesis y conclusiones }\end{array}$ & $60 \%$ & $10 \%$ & $30 \%$ \\
\hline & $\begin{array}{l}\text { Utilización de software para } \\
\text { procesamiento de información y } \\
\text { cálculo numérico }\end{array}$ & $70 \%$ & $20 \%$ & $10 \%$ \\
\hline & & $\mathbf{S}$ & CS & AV \\
\hline \multirow{3}{*}{$\begin{array}{l}\text { Actitud } \\
\text { frente a la } \\
\text { tarea de } \\
\text { aprender }\end{array}$} & Participación en espacios de consultas & $70 \%$ & $30 \%$ & $\begin{array}{c}--- \\
\end{array}$ \\
\hline & Entrega en tiempo y forma & $60 \%$ & $30 \%$ & $10 \%$ \\
\hline & $\begin{array}{l}\text { Evidencias de aprendizaje autónomo } \\
\text { (planteo de nuevos problemas, consulta } \\
\text { ampliada de bibliografía, trabajo en } \\
\text { equipo,....) }\end{array}$ & $50 \%$ & $30 \%$ & $20 \%$ \\
\hline
\end{tabular}




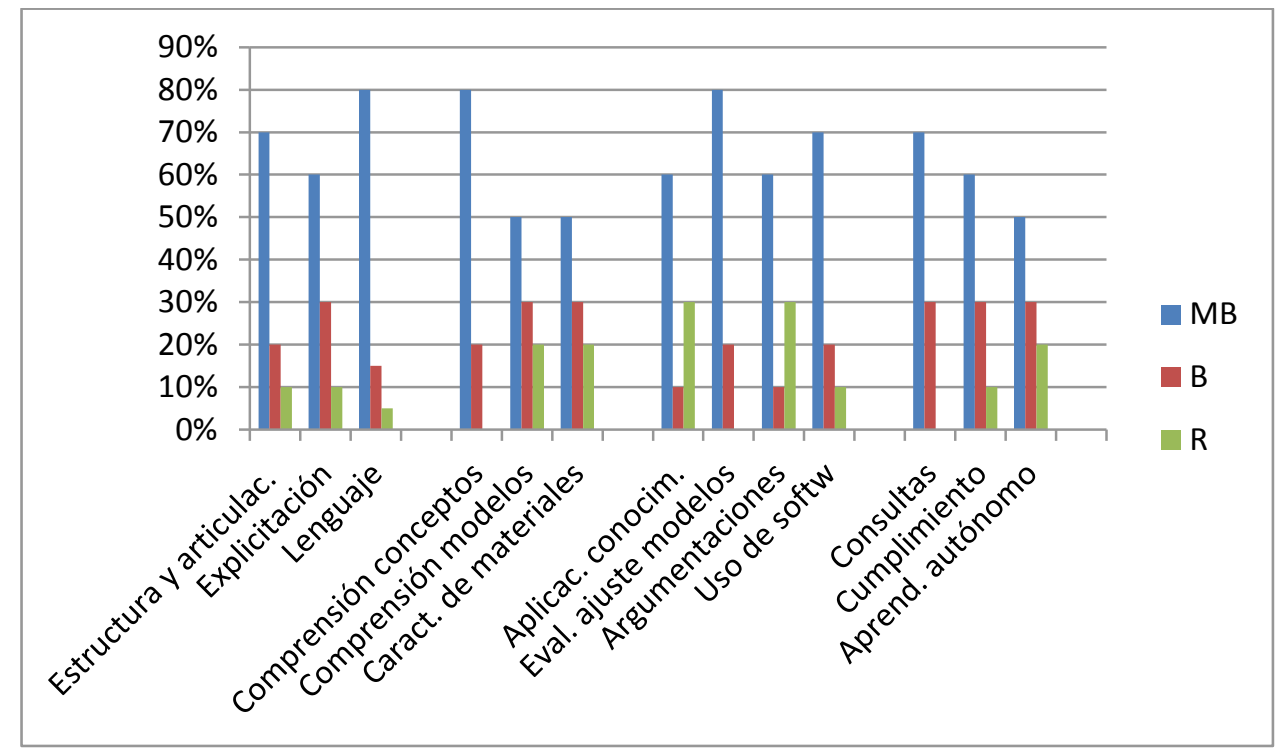

Fig. 2 - Competencias evaluadas a través de informes grupales.

Los resultados obtenidos en la evaluación de los informes grupales, después de la etapa de corrección, muestran que:

- Se ha logrado desarrollar en un nivel muy satisfactorio las competencias consideradas. En efecto, para la mayoría de las competencias que se han evaluado en las producciones grupales, se ha constatado que la suma de los porcentajes de las categorías MB y B supera el $80 \%$.

- Persisten dificultades en el desarrollo de algunas competencias, tales como la comprensión de modelos, la explicación del comportamiento de los materiales, la aplicación del conocimiento teórico en la planificación y desarrollo de una experiencia y el desarrollo de argumentaciones para justificar sus respuestas. Aún después de la etapa de revisión, consultas y corrección se evidencian respuestas no satisfactorias $(\mathrm{R})$ en un porcentaje de aproximadamente $30 \%$.

\section{IV.2. Resultados de la evaluación integradora}

En la tabla 2 se presentan los resultados finales obtenidos en la evaluación integradora con el grupo de alumnos de ingeniería, durante el último ciclo lectivo. Los resultados corresponden a los logros finales del grupo luego de la instancia de 
recuperación de evaluaciones desaprobadas y de la instancia de revisión y corrección de errores.

Se han considerado las categorías Muy Bueno (MB), Bueno (B), Regular (R) y Malo (M) para evaluar el grado de logro de las competencias consideradas. En este caso se presenta también un gráfico de barras que permite visualizar mejor los resultados y comparar los logros obtenidos en cada una de las dimensiones evaluadas (Fig. 3).

Tabla 2 - Resultados generales de la evaluación integradora final.

\begin{tabular}{|l|c|c|c|c|}
\hline \multicolumn{1}{|c|}{ Competencias evaluadas } & MB & B & R & M \\
\hline Comprensión de conceptos, leyes y modelos. & $50 \%$ & $20 \%$ & $20 \%$ & $10 \%$ \\
\hline $\begin{array}{l}\text { Evaluación y estimación del grado de ajuste entre } \\
\text { modelos y sistemas reales. }\end{array}$ & $59 \%$ & $26 \%$ & $11 \%$ & $4 \%$ \\
\hline $\begin{array}{l}\text { Desarrollo de argumentaciones para justificar las } \\
\text { respuestas. }\end{array}$ & $26 \%$ & $41 \%$ & $18 \%$ & $15 \%$ \\
\hline $\begin{array}{l}\text { Aplicación del conocimiento teórico en el diseño, } \\
\text { realización e interpretación de las experiencias. }\end{array}$ & $45 \%$ & $32 \%$ & $18 \%$ & $5 \%$ \\
\hline
\end{tabular}

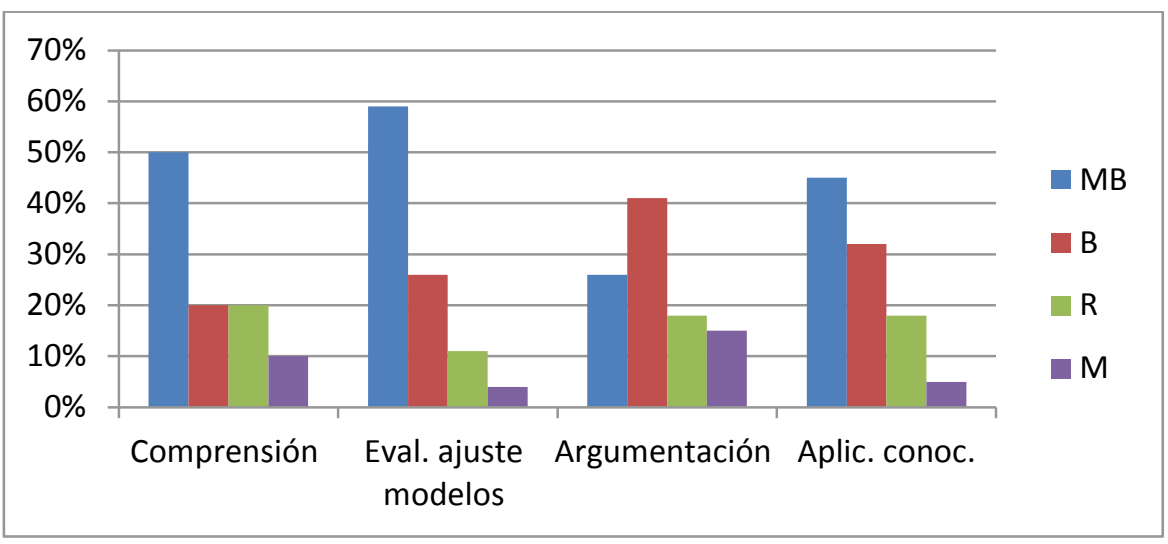

Fig. 3 - Resultados generales de la evaluación integradora final.

Los resultados de la evaluación integradora individual, al finalizar el desarrollo de la propuesta muestran que:

- El porcentaje de respuestas que se evalúan dentro de las categorías B y MB es cercano al $70 \%$ para algunas de las competencias consideradas y mayor que $70 \%$ para otras. Se considera un nivel de logro de competencias cognitivas y 
metodológicas bastante aceptable y se observa que los resultados son coherentes con los obtenidos a través de la evaluación de informes grupales.

- Al igual que en los resultados de las producciones grupales, los alumnos siguen evidenciando dificultades para desarrollar argumentaciones en sus justificaciones y para planificar y realizar una experiencia aplicando su conocimiento aún después de la instancia de corrección.

- El nivel de logro de competencias es levemente inferior al obtenido con los informes y este resultado se interpreta desde las características diferentes de ambos instrumentos. No siempre se puede garantizar que la elaboración del informe grupal sea el producto de la participación de todos los alumnos.

\section{Consideraciones finales}

Integrando las competencias propias de un trabajo de laboratorio a lo largo de todas las actividades, la propuesta se dirige a la comprensión del marco conceptual, al estudio de los modelos que permiten interpretar los resultados experimentales y a la formulación de preguntas que guíen nuevos experimentos. El diseño de las actividades (Fig. $\mathrm{N}^{\circ} 1$ ) supone una serie de objetivos específicos que van a ir pautando las intervenciones del docente que actúa como mediador en este proceso de aprendizaje.

Los objetivos apuntan a integrar permanentemente las competencias básicas para la formación experimental de los ingenieros tales como discriminar e identificar problemas, planificar su resolución a la luz de un marco teórico de referencia, diseñar experimentos plausibles de dar respuesta a los interrogantes planteados, medir, evaluar incertezas y errores sistemáticos, analizar interpretar y explicar resultados, sintetizar conclusiones e identificar aspectos abiertos para futuros abordajes y profundizaciones. Se pone de manifiesto así la importancia del rol del profesor para ayudar a los estudiantes a desarrollar estas habilidades a través del planteo permanente de preguntas, fomentando la participación y el desarrollo de argumentos, mientras trabajan con la guía orientadora.

La metodología de trabajo está sustentada en el marco teórico de Vergnaud, donde el saber y el saber hacer constituyen aspectos indisolubles para el paulatino desarrollo de la conceptualización y el logro de competencias científicas. Se trata de superar visiones que hacen del ámbito del laboratorio sólo un espacio de mediciones acríticas y desarrollo de destrezas para manipulación de instrumentos. Las actividades que se proponen se orientan a generar múltiples 
situaciones de resolución de problemas teórico-experimentales, como espacios de construcción colectiva y de trabajo en equipo.

Los resultados alcanzados, evaluados a través de los informes y las evaluaciones integradoras, pueden considerarse muy alentadores y muestran la fertilidad del marco teórico para orientar la enseñanza, en el ámbito de un laboratorio, hacia el desarrollo de las competencias necesarias en la formación de un estudiante de ingeniería.

La mayoría de los resultados correspondientes a la categoría MB, se interpretan como producto de la instancia de revisión, reflexión y corrección de informes y evaluaciones. En efecto, el marco teórico en que se sustenta esta propuesta considera que durante el aprendizaje pueden producirse avances y retrocesos que implican revisar o repetir una etapa anterior, a los cuales el docente debería atender. Esta instancia se contempla en el esquema de la figura $\mathrm{N}^{\circ} 1$ a través de la doble flecha en cada ciclo de actividades.

Además, los resultados muestran un desarrollo superior en el caso de la capacidad para evaluar el grado de ajuste de modelos y sistemas reales, que en el resto de las competencias evaluadas. Desde el marco teórico, este resultado se justifica por el hecho de que los alumnos vienen realizando prácticas de laboratorio en otras temáticas que les exigen también el desarrollo de esta competencia, por lo cual disponen de un esquema para abordar este tipo de situaciones.

Con respecto al desarrollo de argumentaciones, se observa un logro inferior al resto de las competencias. En este caso se considera que se trata de una habilidad cognitiva que requiere un desarrollo a más largo plazo, por cuanto no sólo está involucrado el uso del lenguaje científico, sino la consistencia en los razonamientos a la hora de formular explicaciones y conclusiones .

Se debe resaltar además, que el ciclo de actividades planteadas en esta propuesta contribuye a profundizar el concepto de resistencia eléctrica. En efecto, la discusión y explicitación de los conceptos que conforman el modelo de Drude, a lo largo de todas las actividades, permite superar interpretaciones erróneas tales como concebir que el aporte a la resistencia eléctrica proviene de los choques con los iones. El modelo de Drude no explicita cuáles son los obstáculos en los choques, es decir, cuáles son los factores que realizan el mayor aporte a la resistencia eléctrica. Se puede discutir con los alumnos la posibilidad de que las colisiones se puedan producir con obstáculos como ser imperfecciones de la red cristalina del sólido, dislocaciones de la misma o las vibraciones de la red cristalina. De esta manera, se puede revisar el concepto de resistencia eléctrica considerando al sólido como un arreglo geométrico periódico de iones positivos y a éstos como entidades dinámicas. 
Por otra parte, al considerar las aplicaciones tecnológicas de los distintos materiales (electrónica, microelectrónica, transporte de energía y/o de información, protección, control de carga etc.) los alumnos se enfrentan con la necesidad de revisar las características de los modelos de conducción, sus supuestos y limitaciones. Estas constituyen situaciones propias de la actividad profesional del ingeniero, cuyo abordaje requiere el conocimiento de la física y promueve la adquisición de las competencias deseadas.

\section{Referencias}

DIAZ J., A. Cambiando la práctica docente en la enseñanza de las ciencias a través de CTS. In: Sala de Lectura CTS+I de la OEI. 2001. Disponible en: $<$ http://www.oei.es/salactsi/acevedo2.htm>.

ZUÑEDA, A. M. Diseño del trabajo de laboratorio con bases epistemológicas y cognitivas: caso del profesorado de física. 2004. Tesis (Doctoral) - Universidad de Burgos, Burgos, España.

ASCHCROFT, N. W.; MERMIN, N. D. Solid state physics. New York: Cornell University, Harcourt College Publisher, 1976. cap. 1, p. 1-30.

AGUEDA, B.; CRUZ, A. Nuevas claves para la docencia universitaria en el Espacio Europeo de Educación Superior. Madrid: Ed. NARCEA, 2005.

BAROLLI, E.; LABURÚ, C.; GURIDI, V. Laboratorio didáctico de ciencias: caminos de investigación. Revista Electrónica de Enseñanza de las ciencias, v. 9, n. 1, p. 88-110, 2010.

BENEITONE, P.; ESQUETINI, C.; GONZALEZ, J.; MALETÁ, M.; SIUFI, G.; WAGEnAAN, R. Final Report ALFA Tuning America Latina Project: Reflections on and outlook for Higher Education in Latin America. 20042007. Disponible en:

$<$ http://tuning.unideusto.org/tuningal/index.php?option=com_docman\&Itemid=19 $1 \&$ task=view_category\&catid=22\&order=dmdate_published\&ascdesc $=$ DESC $>$.

CARRASCOSA, J.; GIL PÉREZ, D.; VILCHES, A. Papel de la actividad experimental en la educación científica. Caderno Brasileiro de Ensino de Física, v. 23, n. 2, p. 157-181, 2006. 
CULLEN, C. El debate epistemológico de fin de siglo y su incidencia en la determinación de las competencias científico tecnológicas en los diferentes niveles de la educación formal. Parte II. Novedades Educativas, n. 62, p. 2, 1996.

FERNÁNDEZ, I.; GIL PÉREZ, D.; VILCHES, A.; VALDÉS, P.; CACHAPUZ, A.; PRAIA, J.; SALINAS, J. El olvido de la tecnología como refuerzo de las visiones deformadas de la ciencia. Revista Electrónica de Enseñanza de las Ciencias, v. 2, n. 3, p. 331-352, 2003.

FOUREZ, G. El movimiento ciencia, tecnología, sociedad (CTS) y la enseñanza de las ciencias. Perspectivas UNESCO, v. XXV, n. 1, p. 27-40, 1995.

GIL PÉREZ, D.; VALDÉZ CASTRO, P. La orientación de las prácticas de laboratorio como investigación: un ejemplo ilustrativo. Enseñanza de las Ciencias, v. 14, n. 2, p. 155-163, 1996.

HODSON, D. Hacia un enfoque más crítico del trabajo de laboratorio. Enseñanza de las Ciencias, v. 12, n. 3, p. 299-313, 1994.

HOFSTEIN, A.; KIND, L. Learning in and from science laboratorios. Second International Handbook of Science Education. USA: Fraser B., Tobin K, Campbell J. Editors - Springer, p. 189-208, 2012.

HOFSTEIN, A.; LUNETTA, V. N. The laboratory in science education: foundation for the $21^{\text {st }}$ century. Science Education, v. 88, p. 28-54, 2004.

ISLAS, S.; PESA, M. Concepción de los profesores sobre el rol de los modelos científicos en clases de física. Revista de Enseñanza de la Física, v. 17, n. 1, p. 43-50, 2004.

MAIZTEGUI, A. et al. Papel de la tecnología en la educación científica: una dimensión olvidada. Revista Iberoamericana de Educación-OEI, n. 28, p.129$155,2002$.

PESA, M.; ISLAS, S.; MEDINA, C.; BRAVO, S. Argumentation in Science Teaching. Science Education Research in South and Latin America. CHARBEL EL-HANI, C.; MORTIMER, E., OTERO, M. R. (Eds.). New York: Routledge. En prensa. 2013. 
POZO, J.; PÉREZ ECHEVERRÍA, M. Psicología del aprendizaje universitario: La formación en competencias. Edic. Morata, 2009.

SALINAS, J. Las prácticas de física básica en laboratorios universitarios. 1996. Tesis (Doctoral) - Universidad de Valencia, España. Disponible un resumen en: <http://www.raco.cat/index.php/ensenanza/article/viewFile/21415/93375>.

SALINAS, J. et al. La formación experimental en Física en los ciclos básicos de carreras de ingeniería. Revista de Ciencias Exactas e Ingeniería, n. 14, p. 48-54, 1998.

SERWAY, R. A.; JEWETT JR., J. Física para ciencias e ingenierías. 6. ed. México: Thomson Learning, 2005. v. II.

TRUMPER, R. The physics laboratory: a historical overview and future perspectives. Science \& Education, v. 18, n. 7, p. 761-774, 2003.

VALDÉZ CASTRO, P.; VALDÉZ CASTRO, R. Características del proceso de enseñanza-aprendizaje de la Física en las condiciones contemporáneas. Enseñanza de las Ciencias, v. 17, n. 3, p. 521-531, 1999.

VERGNAUD, G. Teoria dos campos conceituais. In: NASSER, L. (Ed.). SEMINÁRIO INTERNACIONAL DE EDUCAÇÃO MATEMÁTICA, 1, 2003, Rio de Janeiro. Anais... p. 1-26.

VERGNAUD, G. ¿Por qué la teoría de los campos conceptuales? Infancia y Aprendizaje, v. 36, n. 2, p. 131-161, 2013.

VYGOTSKY, L. Mind in Society. Cambridge: Harvard University Press, 1978.

\section{Agradecimientos}

Los autores agradecen los valiosos aportes realizados por los evaluadores de este trabajo. 


\section{Anexo I: Guía de Actividades}

\section{Relación entre tensión y corriente en materiales eléctricos}

\section{Situaciones problemáticas}

- ¿Cómo se comportan eléctricamente los distintos materiales que Ud. conoce: cerámicos, metales, plásticos etc.?

- ¿Qué modelos explican estos comportamientos diferentes?

- ¿Qué aplicaciones tecnológicas importantes dependen de las propiedades eléctricas de los materiales?

\section{Objetivos de la experiencia}

Estudiar experimentalmente la relación entre tensión y corriente para un conductor metálico y un material semiconductor y estudiar los modelos que explican los resultados obtenidos. Profundizar el concepto de resistencia eléctrica.

\section{Actividades referidas a un conductor eléctrico}

\subsection{Actividades introductorias}

- Diseñe un método que le permita estudiar experimentalmente la relación entre tensión y corriente para un conductor eléctrico.

- Realice el esquema del circuito que va a usar

- Analice fuentes de errores sistemáticos en cada uno de los dos casos. Considere de qué modo se pueden minimizar efectos de calentamiento en el circuito.

- Seleccione con cuidado las características que conviene que tenga cada uno de los elementos que intervienen en el circuito (fuente, amperímetro, voltímetro, reóstato).

\subsection{Actividades de desarrollo. Comportamiento de un metal}

- Mida tensión y corriente en el conductor para distintos valores de la tensión suministrada por la fuente.

- Represente gráficamente los datos obtenidos.

- Controle si los datos admiten un ajuste lineal. En caso afirmativo, explicite la relación funcional entre tensión y corriente. Determine los valores acotados de los parámetros que intervienen. Revise bibliografía e identifique el significado físico de los parámetros. 


\subsection{Actividades de discusión, elaboración de conclusiones y búsqueda de modelos explicativos}

- Analice críticamente sus resultados y describa sus conclusiones considerando:

a) Las incertezas de sus resultados experimentales.

b) Limitaciones del o los métodos elegidos.

c) Explique si puede o no describir a sus resultados como una "Ley de la Física de los materiales"

d) El rango de validez de la relación obtenida.

e) La influencia de las condiciones ambientales en sus resultados.

- Revise en la literatura el modelo teórico de Drude para la conducción eléctrica en metales. ¿Puede explicar los resultados en base a ese modelo? Relacione los parámetros del modelo con las magnitudes medidas en la práctica.

\section{Actividades referidas a un semiconductor}

- Considere ahora un material semiconductor y repita cada una de las actividades referidas al comportamiento de los metales. Ponga especial cuidado en la elección de los instrumentos de medición.

- Analice cualitativamente el modelo de bandas para la conducción eléctrica. En base a la descripción que ofrece el modelo de bandas de energía ¿Puede explicar los resultados en semiconductores utilizando al modelo de Drude? ¿Qué supuestos debe hacer? ¿Puede considerar que los electrones de la banda de conducción del semiconductor usado se comportan como el gas de electrones del modelo de Drude?

\section{Bibliografía básica para el desarrollo del trabajo experimental}

- Errores experimentales, Laboratorio de Física.

- Guía de Apoyo Docente. Laboratorio de Física Experimental

- Fernández y Galloni. Trabajos Prácticos de Física.

- Resnick, Halliday y Krane. Física. Tomo II.

- Sears, Zemansky, Young y Freedman. Física. Volumen 2

- Tipler. Física. Volumen 2. 


\section{Anexo II: Evaluación integradora}

1- En una experiencia realizada en el laboratorio para estudiar el comportamiento de un conductor, un estudiante obtuvo la siguiente tabla de valores correspondientes a la medición de la tensión y la corriente:

\begin{tabular}{|c|c|c|c|c|c|}
\hline V (Volt) & $0,4 \pm 0,1$ & $1,6 \pm 0,1$ & $2,5 \pm 0,1$ & $4,0 \pm 0,1$ & $4,8 \pm 0,1$ \\
\hline I (Amp) & $\begin{array}{l}0,08 \quad \pm \\
0,02\end{array}$ & $\begin{array}{l}0,32 \\
0,02\end{array}$ & $\begin{array}{l}0,51 \\
0,02\end{array}$ & $\begin{array}{l}0,79 \\
0,02\end{array}$ & $\begin{array}{l}0,94 \\
0,02\end{array}$ \\
\hline
\end{tabular}

a) Grafique los resultados obtenidos ¿Considera que el conductor se comporta como un metal? Justifique su respuesta.

b) Evalúe la conveniencia de aplicar método gráfico o analítico para el cálculo de los parámetros de la relación entre V e I, justificando el criterio empleado.

2- ¿Cómo expresaría la ley de Ohm? Diga cuales son verdaderas o falsas y justifique.

a) $\mathrm{V} / \mathrm{I}=\mathrm{R}$, donde $\mathrm{V}$ e I son la tensión aplicada y la corriente eléctrica que circula entre dos puntos de un material, respectivamente.

b) $\mathrm{V} / \mathrm{I}=$ cte., donde $\mathrm{V}$ e I son la tensión aplicada y la corriente eléctrica que circula entre dos puntos de un material, respectivamente.

c) La densidad de corriente es proporcional al campo eléctrico aplicado

d) $\mathrm{R}=\rho \mathrm{l} / \mathrm{S}$, donde $\rho$ es la resistividad, 1 la longitud y $\mathrm{S}$ la sección del material.

3- ¿Como se explica la resistencia eléctrica en el modelo de Drude? Diga cuales son verdaderas o falsas y justifique.

a) Por la dependencia de la velocidad térmica de los electrones con la temperatura.

b) Por la dependencia de la velocidad térmica de los electrones con el campo eléctrico.

c) Por la interacción electrostática entre los electrones de conducción y los iones de la red cristalina que forma el material.

d) Por la dependencia de la velocidad de arrastre (promedio) con el tiempo de relajación.

4- Se dispone de una fuente de alimentación de $15 \mathrm{~V}$ y un amperímetro de alcance 1,5 A con 100 divisiones, clase 1 y resistencia interna $0,1 \mathrm{ohm}$. Se desea medir una resistencia incógnita $R_{x}$ del orden de $10 \Omega$. 
a) ¿Qué características debería tener el voltímetro a utilizar (alcance, apreciación, clase y resistencia interna) a fin de minimizar incertezas en las mediciones?

b) Realice un esquema del circuito que utilizaría para realizar la medición.

c) ¿Cuál es el orden de la potencia que debería ser capaz de disipar la resistencia $\mathrm{R}_{\mathrm{x}}$ ?

d) ¿Qué orden de error total se esperaría cometer en la determinación de $R_{x}$ ? Justifique cuantitativamente todas sus respuestas. 